\title{
INVESTIGACIÓN
}

Recibido: 12/03/2021 --- Aceptado: 15/04/2021 --- Publicado: 24/05/2021

\section{PROBLEMAS ÉTICOS EN LA INVESTIGACIÓN CON NEUROMARKETING: UNA REVISIÓN DE LA LITERATURA}

\section{Ethical issues in neuromarketing research: a literature review}

Dongye Lyu: ${ }^{1}$ Tongji University. China.

donlyu@ucm.es

\begin{abstract}
(D) $8 R^{6}$ Luis Mañas-Viniegra: Universidad Complutense de Madrid. España. $\underline{\text { lmanas@ucm.es }}$
\end{abstract}

\section{Cómo citar el artículo:}

Lyu, D., y Mañas-Viniegra, L. (2021). Problemas éticos en la investigación con neuromarketing: una revisión de la literatura. Vivat Academia. Revista de Comunicación, 154, 263-283. http://doi.org/10.15178/va.2021.154.e1351

\section{RESUMEN}

El avance de la tecnología permite el uso de nuevas herramientas en las investigaciones de una forma más precisa y activa. En los últimos años, ha surgido una nueva interdiscipina compuesta por Neurociencia y Marketing, con cuyas técnicas los investigadores pueden evaluar la eficacia de la estrategia de marketing a través del análisis del procesamiento cognitivo del consumidor. Sin embargo, la aparición del Neuromarketing ha provocado algunas preocupaciones y críticas en relación con la intrusión de la medición fisiológica en el estudio del comportamiento del consumidor. Numerosos investigadores han puesto de manifiesto que el uso de algunas de las herramientas de Neuromarketing, como facial coding o fMRI, pueden causar una pérdida de privacidad personal e, incluso, conducir a la discriminación, estigmatización y coerción de individuos o grupos específicos. De ahí, ha surgido la discusión sobre la ética y la responsabilidad en experimentos de Neuromarketing. A partir de una revisión de la literatura científica, este estudio pretende discutir sobre los problemas éticos en los estudios de Neuromarketing, teniendo en cuenta sus surgimientos, desarrollos y futuros. Este artículo proporciona a los investigadores de neuromarketing una referencia en los dilemas éticos del Neuromarketing y también sienta las bases para la investigación de seguimiento del autor.

\footnotetext{
1 Dongye Lyu: Doctorando en Comunicación Audiovisual, Publicidad y Relaciones Públicas por la Universidad Complutense de Madrid, es asistente de investigación en Tongji University (China).
} 
PALABRAS ClAVE: Neuroética - Neurociencia - Neuromarketing Neurocomunicación - Ética - fMRI - EEG.

\section{ABSTRACT}

The advancement of technology allows the use of new tools in investigations in a more precise and active way. In recent years, composed of Neuroscience and Marketing, a new interdisciplinary subject has emerged, with whose techniques researchers can evaluate the effectiveness of the marketing strategy through the analysis of the consumer's cognitive processing. However, the appearance of Neuromarketing has raised some concerns and criticisms in relation to the intrusion of physiological measurement in the study of consumer behavior. Numerous researchers have claimed that the use of some of the Neuromarketing tools, such as facial coding or fMRI, can cause a loss of personal privacy and even lead to discrimination, stigmatization and coercion of specific individuals or groups. Hence, the discussion about ethics and responsibilities in neuromarketing experiments has arisen. Based on a review of the scientific literature, this study aims to discuss ethical problems in Neuromarketing studies, taking into account their emergence, developments and futures. This article provides neuromarketing researchers with a reference on the ethical dilemmas of neuromarketing and also lays the groundwork for the author's follow-up research.

KEYWORDS: Neuroethics - Neuroscience - Neuromarketing Neurocommunication - Ethic - fMRI - EEG.

\section{INTRODUCCIÓN}

La Neurociencia surgió en la década de 1960 como el estudio interdisciplinar e integrado del cerebro, el comportamiento y el sistema nervioso (Abi-Rached \& Rose, 2010) desde sus cuatro niveles molecular, neuroanatómico, fisiológico y celular (Schimtt, 1970), implicando una revolución tecnológica para la investigación académica y profesional.

Su aplicación específica a las Ciencias Sociales se produjo dentro de los ámbitos de la Neurocomunicación (Cuesta-Cambra, Niño-González, \& Rodríguez-Terceño, 2017) o el Neuromarketing como técnicas de investigación actualmente consolidadas (Morin, 2011), ya sea desde la revisión y reflexión teórica o desde su carácter aplicado a experimentos, cuasiexperimentos o estudios de caso.

El Neuromarketing ha permitido complementar en la rama de conocimiento de las Ciencias Sociales las tradicionales encuestas, focus groups y método Delphi, técnicas con las que los investigadores encontraban la limitación de que los participantes fueran capaces de informar conscientemente sobre las percepciones, actitudes o comportamientos estudiados (Ariely \& Berns, 2010), así como las experiencias sociales (Abi-Rached \& Rose, 2010). Entre las críticas recibidas, destaca la obsesión o

Vivat Academia. Revista de Comunicación. 2021, nº 154, 263-283 
“neuromanía" (Legrenzi \& Umiltà, 2011) de reducir la complejidad de la conciencia, el comportamiento, la cultura y la sociedad humanas a su actividad neuronal, puesto que ello implicaría negar la conciencia y singularidad de los humanos, reconociendo su utilidad para comprender el funcionamiento del cerebro (Tallis, 2011).

Sin embargo, el registro de las reacciones no conscientes de los individuos ante determinados estímulos, que son presentados como finalidad de estudio en el Neuromarketing, han hecho surgir dilemas éticos que necesariamente deben identificarse y valorarse en función de los objetivos de investigación.

\section{OBJETIVOS}

El objetivo general de este estudio es realizar una revisión de la literatura científica sobre Neuroética dentro de la rama de conocimiento de las Ciencias Sociales y, específicamente de la interdisciplina del Neuromarketing.

Los objetivos específicos son:

- Estructurar las tecnologías más utilizadas en la investigación con Neuromarketing y, específicamente, las menos intrusivas.

- Identificar los dilemas éticos que surgen en la investigación con Neuromarketing.

- Revisar la literatura científica en Web of Science que aplique específicamente la Neuroética a la rama de conocimiento de Ciencias Sociales y al Neuromarketing.

\section{METODOLOGÍA}

Esta revisión de la literatura científica destaca la conceptualización del uso de tecnologías poco intrusivas en Neuromarketing, sus problemas éticos y su utilidad para registrar las respuestas no conscientes de los públicos, consumidores y usuarios.

Para delimitar esta revisión, los artículos y libros seleccionados debían cumplir los requisitos de centrarse en esta temática, estar indexados en Web of Science y haber tenido relevancia, en función de la relación entre su fecha de publicación y las citas recibidas. En este sentido, hay que señalar que el Neuromarketing es una interdisciplina que concentra su producción científica en las últimas dos décadas.

A partir de estos criterios, el artículo se estructura en dos grandes bloques: tipos de tecnologías no intrusivas más utilizadas en Neuromarketing, contemplando su historia y fundamento tecnológico e investigador, y dilemas éticos, en tanto que la mayoría son comunes para todas las tecnologías, cuestión que reduce su corpus de manera considerable. 


\section{RESULTADOS}

\subsection{Tecnologías poco intrusivas aplicadas al Neuromarketing}

El estudio del comportamiento del consumidor apenas había prestado atención hasta la llegada del Neuromarketing a la estructura fisiológica interna y la respuesta del cuerpo humano a los estímulos comerciales, de comunicación y de marca. En el pasado, los investigadores tendían a usar modelos como el de "estímulo-respuesta del cuerpo", que vincula la reacción interna inobservable del cuerpo humano con la respuesta externa observable, para explicar el comportamiento del consumidor. Y otros métodos, como el cuestionario de autoinforme, tienden a ser incapaces de capturar la preferencia emocional del consumidor e incluso, conducen, a resultados con sesgos inconscientes (Ohme et al., 2011).

Con el objetivo de cubrir esos espacios en el método de investigación tradicional, los académicos dirigieron su atención a la Neurociencia. En la década de 1970, algunos investigadores propusieron la idea de estudiar la conciencia humana desde un nivel neural (Miller \& Lenneberg, 1978), pero en esa etapa, tan limitada por la tecnología, esta idea era solo un sueño poco práctico. Finalmente, desde la década de 1990, la mayor maduración de las imágenes del cerebro y otras tecnologías en el campo de la Neurociencia han hecho que esas técnicas sean ampliamente utilizadas en la investigación de las Ciencias Sociales, como la Psicología, la Economía o la Educación (Madan, 2010). Al mismo tiempo, estas tecnologías avanzadas también se aplicaron en el campo de la investigación de Marketing y Comunicación.

Es a partir de 2002 cuando comienza a emerger rápidamente y a alcanzar su credibilidad actual (Morin 2011). En 2004, el equipo de investigación de McClure utilizó un diseño más complejo para replicar un famoso experimento (Pepsi paradox) realizado por PepsiCo en la década de 1970, que se considera la marca que impulsó el Neuromarketing. Los resultados indican que la información de la marca afecta a los consumidores a través de actividades cognitivas de alto nivel, momento en el cual el procesamiento sensorial de bajo nivel es reemplazado por procesos cognitivos de alto nivel. Desde esta etapa, el Neuromarketing se considera como una interdisciplina compuesta de Neurociencia y Marketing (Braidot, 2009; Santesmases, 2009). Desde 2010, la tendencia actual es tratar de entender el comportamiento y necesidades de consumidores desde la teoría y las herramientas de las Neurociencias aplicadas al Marketing.

El propósito de la investigación de la Neurociencia cognitiva es comprender el mecanismo de trabajo del cerebro. Las herramientas de investigación de la Neurociencia cognitiva muestran y presentan principalmente la estructura y función del cerebro a través de los principios de la Física y la Bioquímica modernas. Al mismo tiempo, algunos indicadores fisiológicos como el movimiento de los ojos, dilatación de la pupila, latido del corazón, presión arterial o el flujo sanguíneo también son el foco de atención. De hecho, las herramientas de Neurociencia cognitiva comúnmente utilizadas incluyen seguimiento ocular (eye-tracking), 
tomografía de emisión de positrones (PET), electroencefalograma (EEG), magnetoencefalograma (MEG), imagen por resonancia magnética funcional (fMRI), respuesta galvánica de la piel (GSR/EDA), prueba implícita (IAT), codificación facial (facial coding) ... Las tecnologías más utilizadas en investigación de mercados se pueden dividir en tres categorías principales en función de la tecnología aplicada, que son piscométrica (IAT), biométricas (codificación facial, seguimiento ocular, GSR) y neurométricas (EEG, fMRI). Además, según sus tipos de método de medición, las herramientas neurométricas también pueden dividirse en dos categorías, que son herramientas para medir los cambios en la actividad neuronal eléctrica (EEG, MEG) y herramientas para medir los cambios en la actividad neuronal metabólica (fMRI, PET) (Ahlert et al., 2006; Harris et al., 2018).

En términos generales, la investigación de Marketing se enfoca principalmente en cuatro temas, incluyendo Marca, Precio, Producto y Comunicación, y la investigación de Neuromarketing no es una excepción (Yan, 2011). En los últimos años, varios autores (Zaltman \& Kosslyn, 2000; Kenning \& Plassmann, 2005; Kenning, Plassmann, \& Ahlert, 2007; Mostafa, 2012) han proporcionado evidencias y explicación específica sobre el uso de herramientas de Neuromarketing en sus investigaciones. Aunque recientemente la mayor parte de las investigaciones de Neuromarketing ha utilizado predominantemente fMRI para medir la activación cerebral en respuesta a estímulos de marketing y experimentos de toma de decisiones (Rilling \& Sanfey, 2011; Smidts et al., 2014), existen muchas otras opciones de herramientas disponibles, que incluyen GSR, seguimiento ocular, codificación facial o EEG. Sobre este aspecto, son muchos los autores (Ohme et al., 2009; de Giraldi \& de Oliveira, 2014; Venkatraman et al., 2015; Harris et al., 2018) que han contribuido a proporcionar una descripción general y una explicación de todas las herramientas de Neurociencia utilizadas en la investigación de Marketing y Comunicación.

\subsection{1. fMRI - fNIRS}

La resonancia magnética funcional es una tecnología neurocientífica no invasiva que utiliza ondas de radio y campos magnéticos para medir los cambios metabólicos (consumo de energía) del flujo de oxígeno en sangre. La fMRI registra señales y variaciones de la actividad dependiente del nivel de oxígeno en sangre en diversas regiones del cerebro de interés. Por tanto, es excelente para identificar la ubicación específica del cerebro a una resolución muy alta, aunque su resolución temporal es baja. Además, la fMRI muestra los cambios mediante la presentación de áreas coloridas superpuestas del cerebro en imágenes en 3D y permite visualizar las estructuras del cerebro en detalle. Sin embargo, aparte de su alto coste, la fMRI también tiene varias limitaciones. En primer lugar, dicha tecnología requiere que el sujeto se ubique en el interior de una máquina en forma de tubo, permaneciendo inmóvil y, en consecuencia, el entorno experimental puede generar ansiedad claustrofóbica e, incluso, influencia en todo el resultado. Además, la fMRI no puede registrar ciertos procesos cognitivos porque la activación cerebral tiene un impacto muy pequeño en los niveles de oxígeno en sangre detectables por fMRI (Kwong et al., 1992; Sanie \& Chalmers, 2007). A pesar de ello, la investigación con Neuromarketing, 
sobre todo referida a la toma de decisiones del consumidor, se ha realizado predominantemente con la fMRI debido a su precisión de la actividad cerebral y existe una amplia literatura científica que reafirma su importancia para identificar la estructura neuronal, así como ubicar la zona que puede influir la toma de decisiones (McClure et al, 2004; Deppe et al., 2007; Knutson et al., 2007; Hare et al., 2008; Grosenic et al., 2008; Santos et al, 2011; Levy \& Glimcher, 2012; Solnais et al., 2013; Smidts et al., 2014; Plassmann et al., 2015).

Por todo ello, en Neuromarketing es cada vez más habitual el uso no invasivo de la espectroscopia funcional de infrarrojo cercano (fNIRS), técnica basada en el consumo de oxígeno en el cerebro a través de las células sanguíneas, registrando la activación que se produce en la corteza cerebral con alta resolución temporal, aunque con baja resolución espacial y menor profundidad que fMRI. Sus equipos permiten la movilidad, son menos costosos y menos intrusivos para un estudio del consumidor (Hupper et al., 2017). En consecuencia, puede clasificarse a fNIRS dentro de las técnicas de neuroimagen y su adecuación a los estudios de Neuromarketing hace que compita habitualmente con los equipos de EEG, descritos a continuación.

\subsubsection{EEG}

El electroencefalograma (EEG) es una de las herramientas más utilizadas en la investigación de Neuromarketing, después de fMRI. La tecnología permite registrar la actividad cerebral en muchas circunstancias y también los datos de actividad eléctrica de las capas superficiales de la corteza. Según la literatura existente, el Dr. Hans Berger realizó la primera grabación de EEG en un experimento humano en 1924 (Ciorciari, 2012).

El EEG se utilizó por primera vez en la investigación publicitaria de Krugman (1971) para medir la sincronización de ondas cerebrales y arrastre en respuesta a los estímulos publicitarios. A partir de la década de 1980, el EEG comenzó a usarse regularmente para la investigación publicitaria. Los primeros estudios han descubierto la utilidad del EEG para comprobar cómo la publicidad se codifica en la memoria a largo plazo y el procesamiento cognitivo-emocional, así como las respuestas a los estímulos publicitarios, incluyendo el reconocimiento y recuerdo no consciente (Ohme et al., 2009). Los principales progresos en los registros de EEG ocurrieron con los avances tecnológicos (Ciorciari, 2012). Como técnica no invasiva, el EEG mide los cambios de la actividad neural en la corteza, por ejemplo, el EEG registra y mide la frecuencia de las corrientes eléctricas del cerebro y los cambios en el voltaje a través de electrodos colocados en el cuero cabelludo (Ahlert et al., 2006; Ohme et al., 2009; Banich \& Compton, 2010; Ohme et al., 2011; Ciorciari, 2012). Registrando la variación eléctrica de las ondas del cerebro (Núñez \& Srinivasan, 2006), los electrodos pueden funcionar como canales individuales, que se unen al hueso mastoideo (como un sitio inactivo) y funcionan como referencia (línea de base) para comparar la actividad eléctrica de los otros sitios de electrodos. Los sistemas de matriz de alta densidad permiten promediar la actividad de un electrodo a los otros sitios de electrodos (Banich \& Compton, 2010). Usando electrodos (sensores) 
conectados al cuero cabelludo, los investigadores pueden agregar y registrar potenciales sumados de actividades neuronales (Ahlert et al., 2006; Sanie y Chambers, 2007). Los cinco anchos de banda de frecuencia de EEG principales son delta $(\delta, 0.5-4 \mathrm{~Hz})$, theta $(\theta, 5-7 \mathrm{~Hz})$, alfa $(\alpha, 8-14 \mathrm{~Hz})$, beta $(\beta, 15-30 \mathrm{~Hz})$ y gamma $(\gamma$, 30-50 Hz). El ancho de banda neuronal varía con los tipos de exposición y procesamiento de estímulos cognitivos y sensoriales. Las diferentes frecuencias indican diferentes ondas cerebrales de diferentes longitudes (Ohme et al., 2011) y cada ancho de banda de frecuencia está asociado con un rango de potencia (Ciorciari, 2012).

Aparte de su facilidad de uso y coste reducido con respecto a la fMRI, su principal ventaja es su alta resolución temporal, que puede registrar actividad neuronal en milisegundos y submilisegundos, por lo que los investigadores pueden detectar las actividades cerebrales con estímulos que cambian rápidamente (Ahlert et al., 2006). Además, el EEG permite comparaciones entre los hemisferios izquierdo y derecho, la medición de las tendencias de aproximación (dominancia del hemisferio izquierdorespuestas emocionales positivas) o retirada (dominancia del hemisferio derechorespuesta emocional negativa). Sin embargo, el EEG tiene una resolución espacial muy limitada (según el número de electrodos, a partir de dos) y los resultados del EEG no ofrecen datos de las partes más internas del cerebro, ya que la medición se realiza a nivel del cuero cabelludo (Ciorciari, 2012). Además, como la conductividad eléctrica puede diferir de una persona a otra, es difícil recuperar la ubicación exacta de cada señal.

Pese a todo ello, actualmente, debido a su fiabilidad en términos de medición de respuestas neuronales de mensajes dinámicos, el EEG se utiliza con frecuencia en la investigación publicitaria para medir las respuestas de los consumidores en términos de valencia o engagement, relevancia, atención cognitiva o el pronóstico de la elección de marca (Rothschild et al., 1986; Vecchiato et al., 2010; Gountas et al., 2014; Pynta et al., 2014; Telpaz et al., 2015).

\subsubsection{Eye tracking}

El seguimiento ocular (eye tracking) permite estudiar el comportamiento y la cognición identificando hacia dónde están mirando los sujetos sin medir sus actividades cerebrales. El seguimiento de los ojos se considera información más precisa que los auto-informes, como muestra la investigación en la que se demostró que el informe del sujeto no siempre coincide con la visualización real medida. (Zurawicki, 2010; O'Connell et al., 2011)

El análisis del movimiento ocular fue documentado en primer lugar por Nixon (1924) y Karslake (1940) en respuesta a la visualización de estímulos publicitarios impresos. Más tarde, los experimentos de movimiento ocular se utilizaron gradualmente como pruebas para analizar los procesos de toma de decisiones (Russo, 1978; Wedel \& Pieters, 2008) y la mejora de las tecnologías de seguimiento 
ocular han contribuido con una gran cantidad de resultados útiles de investigación con Neuromarketing en la década de 1990 (Wedel \& Pieters, 2008).

Generalmente, hay tres tipos básicos de movimientos oculares: sacádicos, de fijación o seguimiento y de convergencia (Andreassi, 2007). En lugar de pausas, las sacádicos son movimientos de fijaciones oculares que ocurren en rápida sucesión (las fijaciones ocurren durante aproximadamente 250-1,000 ms, mientras que el movimiento sacádico oscila entre 20 y $100 \mathrm{~ms}$ ). También puede producirse la fijación y seguimiento de un objeto en movimiento y los movimientos de convergencia, que son oscilaciones involuntarias como barridos horizontales lentos y retornos rápidos a la posición original del ojo (Andreassi, 2007). En la literatura existente, el tiempo de fijación y el número de fijaciones son las medidas de atención hacia las áreas de interés (AOI) más utilizadas (Jacob y Karn, 2003). Según la investigación de Zhang (2015), el movimiento de los ojos se puede grabar para objetos dinámicos en anuncios de vídeo, ya que se puede rastrear la atención visual de los espectadores para cada AOI. El número de fijaciones y el tiempo medio de cada fijación proporcionan una medida de la profundidad a la que se procesa la información dentro de un anuncio. Un mayor tiempo de fijación y menos fijaciones representan un procesamiento más detallado (Hosrtmann et al., 2009).

Existen cuatro tipos de datos que se usan frecuentemente con el eye tracking, que son: la duración de la primera fijación, latencia de la primera fijación, cantidad de fijaciones y el tiempo total de contacto en cada AOI (Ho, 2014). La tecnología del eye tracking utiliza las categorías mencionadas para analizar el problema y luego muestra los resultados de las pruebas al proporcionar gráficos intuitivos, mapas de calor (heat maps), diagramas de la mirada (gaze plots) y rutas de exploración (scan paths) en respuesta a los estímulos presentados.

Debido al avance en la tecnología, el dispositivo eye tracker moderno facilita la evaluación de la atención visual, porque puede grabar el movimiento ocular de los consumidores en condiciones de exposición natural, con una variedad de estímulos diferentes. Como resultado, la tecnología ahora se usa ampliamente en la investigación de publicidad, sea estática, dinámica o visualizada en interior o exterior, la usabilidad e interacción de apps y páginas web, el packaging de los productos o los logos de marcas, por poner algunos ejemplos.

Aunque los resultados dependen mucho de las condiciones del ojo de los sujetos, el eye tracking, como un método no invasivo, todavía tiene varias ventajas. La tecnología es capaz de detectar la atención espacial y el equipo es conveniente y fácil de llevar. En comparación con otros métodos, el eye tracker puede apuntar los cambios en la dilatación de la pupila y la velocidad de frecuencia de parpadeo, que proporcionan información precisa sobre la participación en el procesamiento imágenes y en el grado de excitación. Además, el seguimiento ocular se utiliza a menudo como un complemento útil de otras herramientas de Neuromarketing, por ejemplo, cuando pretendemos analizar el comportamiento del consumidor en una 
tienda llena de productos, lo mejor será combinar el EEG con seguimiento ocular para saber exactamente qué está produciendo las reacciones cerebrales.

\subsubsection{Facial coding}

La codificación de las emociones a través de las expresiones faciales, facial coding o electromiografía facial, identifica y mide micro-expresiones que codifican las reacciones no conscientes sobre la base del movimiento voluntario o involuntario los músculos faciales. La teoría contempla 6 emociones básicas: ira, desagrado, alegría, sorpresa, tristeza y miedo, si bien se han llegado a detallar hasta en 27 emociones específicas. Las expresiones faciales son espontáneas y proporcionan datos en tiempo real, pero se basan en una cierta subjetividad para decidir cuándo se ha producido una acción o cuándo se cumple con los requisitos mínimos para la codificación.

Los músculos faciales más comúnmente registrados son el corrugador, el orbicular, y el cigomático. La ubicación de estos músculos es la siguiente: el cigomático (mejilla, por encima de la comisura de la boca), el corrugador (ceja sobre el puente de la nariz), y el orbicular (párpado inferior) (Cacioppo et al., 1986; Bolls et al., 2001). El movimiento del músculo cigomático es voluntario, asociado con la sonrisa y la emoción positiva, mientras que el corrugador está asociado con el ceño fruncido y la emoción negativa (Cacioppo et al., 1986). Las respuestas del corrugador y el orbicular son involuntarias y las grabaciones de estos movimientos musculares miden las respuestas emocionales no conscientes a las comunicaciones a las que se exponen los sujetos (Ohme et al., 2011).

La investigación ha demostrado que la codificación facial ofrece información valiosa para medir respuestas emocionales positivas y negativas a estímulos publicitarios. (Bolls et al., 2001), si bien el software disponible actualmente registra mayoritariamente emociones neutras ante la dificultad de diferenciar los gestos faciales no evidentes. La electromiografía facial se ha utilizado para evaluar una variedad de estímulos de Marketing, como la valencia emocional positiva o negativa mientras se escuchan anuncios de radio (Bolls et al., 2001) y respuestas emocionales al material publicitario (Ohme et al., 2011).

\subsubsection{Respuesta galvánica de la piel (GSR)}

La actividad electrodérmica (EDA) registra los cambios en la conductividad de la piel frente a la excitación emocional (emotional arousal). Cuando el cuerpo humano cambia los síntomas físicos, como la contracción y expansión de los vasos sanguíneos epidérmicos de la piel o la secreción de las glándulas sudoríparas debido a cambios emocionales, provoca un cambio correspondiente en la resistencia de la piel y forma una reacción cutánea eléctrica (respuesta galvánica de la piel, galvanic skin response o GSR). Descubierta por Feré (1881), la GSR se puede utilizar para medir la conductancia de la piel y la excitación del sistema nervioso autónomo en respuesta a estímulos de marketing sensoriales y cargados de emociones (Ohme et al., 2009). 
La medición de la conductancia de la piel en respuesta a estímulos publicitarios se utilizó por primera vez durante la década de 1970 (Kroeber-Riel, 1979; Ahlert et al., 2006). Además de ser un indicador de la función del sistema nervioso simpático, las señales eléctricas de la piel también se pueden usar como indicadores de la activación del cerebro y los niveles de excitación, que se pueden utilizar para medir el nivel de excitación emocional (LaBarbera y Tucciarone, 1995). Normalmente, los cambios en la piel y el sudor se pueden medir mediante una banda simple con un electrodo envuelto alrededor de la punta del dedo, lo que hace que sea muy fácil y no invasiva su utilización gracias a una cómoda pulsera.

La señal de respuesta eléctrica de la piel es una de las señales fisiológicas más utilizadas en el campo de la investigación del reconocimiento de emociones. Muchas consultoras de Neuromarketing incorporan el GSR habitualmente a sus metodologías para detectar el impulso de compra en los sujetos estudiados. Dicha tecnología puede medir la respuesta del sistema nervioso autónomo, que los consumidores descontrolan en gran medida (LaBarbera y Tucciarone, 1995). Normalmente, el GSR se usa con frecuencia junto con otros datos biométricos como EEG y fMRI, además del eye tracking, porque una de las principales limitaciones de GSR es que no puede detectar la valencia positiva o negativa del nivel de excitación emocional (Ohme et al., 2009).

\subsection{Dilemas éticos del Neuromarketing}

La interdisciplina del Neuromarketing tiene como objetivo comprender, predecir, cambiar o, al menos, evaluar, el comportamiento del consumidor en función de las reacciones que experimenta el cerebro ante los estímulos de carácter comercial (Ariely \& Berns, 2010; Spence, 2019). Las empresas, por su parte, utilizan esta información para influir en el consumidor al lanzar al mercado productos/servicios, campañas publicitarias o estímulos en el punto de venta que favorezcan su actividad comercial. Esta finalidad puede extenderse a otros comportamientos sociales e, incluso, morales (Greene, 2013).

A partir de estos objetivos, se ha puesto de manifiesto una serie de peligros y riesgos que algunos países han optado por regular desde el punto de vista legal y desde la autorregulación que ejercen las asociaciones científicas y profesionales. En este contexto, surge la Neuroética como la intersección de la Neurociencia y la Bioética con el análisis de los trabajos desde un enfoque filosófico, de humanidades, legales y políticos (Illes, 2007). A medida que la Neurociencia avanza a la hora de comprender, predecir e influir sobre la mente humana, van surgiendo conjuntos más amplios de problemas éticos (Farah, 2010). A su vez, la Neuroética genera una mayor complejidad en las ya de por sí complejas investigaciones de Neurociencia, a pesar de su imprescindible aplicación en una organización guiada desde los principios de la actuación responsable (Illes, 2007). 
La privacidad es una de las cuestiones íntimamente ligadas a la libertad cognitiva (Sententia, 2013). La monitorización no invasiva de la actividad cerebral de equipos como fMRI o EEG se ha complementado con otros que pueden medir aspectos de la fisiología periférica, como el eye tracking o la GSR, siendo los más utilizados en Neuromarketing (Stanton et al., 2017). El consentimiento informado transmite a los participantes cuál es el objetivo del estudio, los datos que se recogen y los riesgos que implica su participación, por los cuales cualquier sujeto puede decidir interrumpir y abandonar la investigación sin necesidad de alegar causa alguna para ello, de conformidad con la denominada Declaración de Helsinki.

Sin embargo, los individuos deben renunciar voluntariamente a parte de su privacidad en aras de los resultados de la investigación. Es obvio que no podrá realizarse un análisis de las microexpresiones faciales si no se graba el rostro del individuo o que no podrán identificarse el tipo de emociones sin la imagen de su activación cerebral. Si bien cabe la posibilidad de eliminar estas grabaciones e imágenes una vez que se han exportado los datos al software estadístico, se dificultaría la posterior revisión por pares de la comunidad científica, cuestión que podría invalidar la validez de los resultados de investigación obtenidos.

Esta incidencia de la privacidad incrementa los riesgos cuando la investigación está basada en las imágenes del cerebro. La neuroimagen o resonancia magnética funcional (fMRI) registra la función cerebral a partir de los cambios que se producen en la oxigenación sanguínea en cada región cuando el individuo se expone a los estímulos presentados (Moseley \& Glover, 1995). Esta técnica de imagen cerebral ha sido identificada en el $60-70 \%$ de los estudios empíricos de Neurociencia aplicada a la toma de decisiones (Kable, 2011). El principal dilema ético identificado es que permite recopilar información sobre los rasgos psicológicos de un individuo, ya sea sobre la personalidad, actitudes o inteligencia, aunque de forma muy limitada, puesto que las imágenes del cerebro no permiten visualizar el pensamiento humano como tal, solo correlaciones a partir de sus funciones cognitivas (Illes, 2007).

Sin embargo, las imágenes del cerebro, tanto en el caso de fMRI como de EEG o fNIRS, con menor coste y resolución espacial (De-Martino et al., 2006), podrían obtenerse con consentimiento para una finalidad y ser posteriormente analizadas con otros propósitos (Farah et al., 2010). Algunos autores ponen como ejemplo los sesgos implícitos, como un estudio sobre percepción del rostro humano que posteriormente pudiera ser utilizado para comprobar la actividad cerebral evocada con respecto a actitudes raciales no conscientes (Phelps, 2000). De igual modo, es posible reconstruir un estímulo visual a partir de la actividad de la corteza visual del cerebro o identificar las correlaciones neuronales de la mentira (Farah et al., 2014), siempre de manera limitada, por lo que la decodificación plantea un nuevo dilema en relación con la privacidad de la actividad cerebral (Roskies, 2015).

La literatura científica plantea también problemas éticos en la propia definición de la investigación con Neuromarketing, tanto desde el punto de vista de la 
planificación de los experimentos como de la selección de las muestras y la validez de los resultados, a menudo exagerando los hallazgos sin incidir en sus limitaciones.

La recreación del entorno simulado en un laboratorio implica aislar a los participantes de la experiencia real de consumo (Spence, Piqueras-Fiszman, 2014) y, en consecuencia, hay que tomar los resultados como una emulación que impide que la respuesta no consciente sea idéntica de la que se produciría en la realidad (Mileti et al., 2016) y, en consecuencia, dificulta la predicción de los comportamientos, pese a su utilidad para acercarse a la comprensión de las causas por las que se produce (Berns et al., 2010). Por ejemplo, en un entorno simulado es habitual que se produzca una mayor atención hacia los estímulos presentados, ya que los sujetos desean colaborar con la investigación con su mejor disposición. Esta cuestión no es ajena a otras técnicas tradicionales de investigación en Marketing y Comunicación, como el hecho de que los sujetos en ocasiones traten de adecuar sus respuestas a lo que se espera o a lo socialmente aceptado.

La representatividad de las muestras de reducido tamaño que se emplean en las investigaciones de Neurociencia, habitualmente entre 20 y 60 sujetos por grupos (Mañas-Viniegra et al., 2020a), es otro de los dilemas que se han puesto de manifiesto, si bien es aplicable a otras técnicas clásicas, como los focus groups. También es reseñable que la mayor parte de estas investigaciones se lleva a cabo en países occidentales con participantes con educación superior (Jones, 2010), con un sesgo cultural que no contempla las diferencias entre sociedades más colectivas frente a otras más individualistas en los comportamientos de los consumidores (Tiggemann \& Zaccardo, 2016; Mañas Viniegra et al., 2020b).

Quizás existirían más estudios de tipo comparativo o cross-cultural si la tecnología fuese más accesible. En efecto, como los equipos de Neuromarketing tienen un elevado coste y algunos de ellos tienen una disponibilidad limitada en países emergentes (Ariely \& Berns, 2010), no resulta sencillo encontrar laboratorios preparados para colaborar en varios países simultáneamente, pese a que han proliferado los equipos de bajo coste. El problema principal de los equipos de bajo coste, que han permitido su acceso a equipos de investigadores sin financiación, es que se corre el riesgo de que no gocen de una adecuada sensibilidad para el registro de los datos o que se produzcan falsos positivos -o, por el contrario, insuficiente registro- en la activación de regiones cerebrales (Illes, Kirschen, \& Gabrielli, 2003).

$\mathrm{Si}$ precisamente los costes de los equipos son una de las limitaciones de la investigación con Neuromarketing, podría producirse el riesgo de que equipos de investigación infrafinanciados intentasen optimizar en exceso el tiempo de realización de los experimentos. Por ejemplo, una adecuada calibración de los equipos requiere un tiempo necesario con cada participante hasta que los registros del eye tracker se ajusten a la fijación de la atención hacia el área de interés del estímulo que efectivamente se está mirando. 
En última instancia, la finalidad del Neuromarketing desde un punto de vista comercial al perseguir la adaptación -manipulación para sus detractores- de los productos, la comunicación, la estimulación en el punto de venta... conforme a los deseos, gustos y preferencias de los públicos puede ser considerada como una forma sutil de interferir en la autonomía del consumidor (Stanton et al., 2014), tanto en las creencias, estados de ánimos y el propio proceso de toma de decisiones y comportamiento del consumidor. Si bien existe el riesgo de que este tipo de investigaciones pueda acrecentar el trastorno de compra compulsiva, también puede utilizarse para su detección y evitar la utilización de estímulos que puedan potenciarlo (Stanton et al., 2017).

Para finalizar, no hay que olvidar que la Neuroética también aplica a la publicación de los resultados obtenidos en las investigaciones, especialmente en la búsqueda de una mayor financiación en su divulgación a través de los medios de comunicación, en tanto que existe el riesgo de que los hallazgos sean tomados al pie de la letra con un enfoque que aliente el entusiasmo o el miedo, no presentando interpretaciones alternativas o sus limitaciones a un público con un conocimiento medianamente informado (Racine et al., 2010). Es más, existen estudios que consideran más persuasivos los estudios presentados con coloridas imágenes cerebrales, incluso en audiencias científicas especializadas, aunque se ha rebajado la fuerte influencia que presentaron los estudios de mayor antigüedad (McCabe \& Castel, 2008; Farah \& Hook, 2013).

\section{CONCLUSIONES}

Considerando que el Neuromarketing simula en el laboratorio un entorno natural para realizar el estudio de públicos, consumidores y usuarios, se prima el uso de equipos no invasivos ni intrusivos -como eye tracking, EEG, fNIRS, GSR o facial coding- $\mathrm{y}$, preferiblemente, que permitan la movilidad, lo que provoca que el detalle de los resultados sea inferior a otros estudios más propios de Ciencias de la Salud, con un mayor uso de fMRI y otros equipos más precisos. Sin embargo, en el estudio de la actividad cerebral aplicado a consumidores y seguidores de las marcas, las correlaciones no implican causalidad (Vul et al., 2009; Plassman et al., 2015), de ahí la importancia de combinar los datos de atención con los de emoción para llegar a la percepción cognitiva y la toma de decisiones. En definitiva, la capacidad de predecir estas cuestiones a partir de métodos de Neurociencia se sitúa entre el 70\% y el $80 \%$ (Varan et al., 2015), siendo en el área académica del Neuromarketing donde se puede proporcionar la información más relevante (Spence, 2019).

La mayoría de investigadores coincide en señalar, con independencia del momento en las últimas dos décadas (Nill \& Schibrowsky, 2007; Stanton, SinnottArmstrong, \& Huettel, 2017), que los dilemas y riesgos éticos que afectan al Neuromarketing no son nuevos y pueden atribuirse al marketing tradicional, mientras que se han magnificado los poderes que la tecnología todavía no permite de un modo realista, cuestión que evidencia el crecimiento de las críticas que recibe esta técnica de investigación (Plassmann et al., 2012), que a menudo confunde la

Vivat Academia. Revista de Comunicación. 2021, nº 154, 263-283 
influencia con el control, pudiendo producirse esta influencia sin necesidad de haber utilizado técnica de Neuromarketing alguna, como históricamente han hecho los estudios del comportamiento del consumidor. Que el consumidor no sea consciente de las variables que influyen en su elección en el punto de venta no implica que su decisión no sea libre.

Sin embargo, resulta esencial que se fomente la autorregulación para complementar los ámbitos más precisos y la cauística que la normativa legal no puede delimitar, para los cuales la Neuroética desempeña un papel fundamental en cuestiones como el consentimiento informado, la privacidad, el tamaño y diversidad adecuados de las muestras, la rigurosidad en el análisis de los resultados en un entorno simulado o la responsabilidad en la difusión de unos hallazgos que no minimicen las limitaciones.

\section{REFERENCIAS}

Abi-Rached, J. M., \& Rose, N. (2010). The Birth of the Neuromolecular Gaze. History of the Human Science, 23(1), 11-36. https:/ / doi.org/10.1177/0952695109352407

Ahlert, D., Kenning, P., \& Plassmann, H. (2006). A window to the consumer's mind: Application of functional brain imaging techniques to advertising research. In $S$. Diehl, \& R. Terlutter (eds.), International Advertising and Communication (pp. 163178). Springer.

Andreassi, J. L. (2007). Psychophysiology. Human behavior E physiological response (5th. ed.). Lawrence Erlbaum.

Ariely, D., \& Berns, G. S. (2010). Neuromarketing: The hope and hype of neuroimaging. Business. Nature Reviews Neuroscience, 11, 284-292. https://doi.org/10.1038/nrn2795

Banich, M. T., \& Compton, R. (2010). Cognitive Neuroscience. Cengage Learning.

Berns, G. S., \& Moore, S. (2012). A neural predictor of cultural popularity. Journal of Consumer Psychology, 22, 154-160. https://doi.org/10.1016/j.jcps.2011.05.001

Bolls, P. D., Lang, A., \& Potter, R. F. (2001). The effects of message valence and listener arousal on attention, memory, and facial muscular responses to radio advertisements. Communication Research, 28(5), 627-651. https:// doi.org/10.1177/009365001028005003

Braidot, N. (2009). Neuromarketing. Gestión 2000.

Cacioppo, J. T., Petty, R. E., Losch, M. E., \& Kim, H. S. (1986). Electromyographic activity over facial muscle regions can differentiate the valence and intensity of 
affective reactions. Journal of Personality and Social Psychology, 50(2), 260-268. https://doi.org/10.1037/0022-3514.50.2.260

Ciorciari, J. (2012). Bioelectrical signals: The electroencephalogram. In A. Wood (ed.), Physiology, biophysics and biomedical engineering (chapter 20). CRC Press. https://doi.org/10.1201/b11558

Cuesta-Cambra, U., Niño-González, J. I., \& Rodríguez-Terceño, J. (2017). The cognitive processing of an educational app with EEG and eye tracking. Comunicar, 52(3), 41-50. https://doi.org/10.3916/C52-2017-04

De-Martino, B., Kumaran, D., Seymour, B., \& Dolan, R. J. (2006). Frames, biases, and rational decision-making in the human brain. Science, 313(5787), 684-687. https:// doi.org/10.1126/science.1128356

Deppe, M., Schwindt, W., Pieper, A., Kugel, H., Plassmann, H., Kenning, P., \& Ringelstein, E. B. (2007). Anterior cingulate reflects susceptibility to framing during attractiveness evaluation. Neuroreport, 18(11), 1119-1123. https://doi.org/10.1097/WNR.0b013e3282202c61

Farah, M. J. (2010). Neuroethics: An introduction with readings. The MIT Press.

Farah, M. J., \& Hook, C. J. (2013). The seductive allure of seductive allure. Perspectives on Psychological Science, 8, 88-90. https://doi.org/10.1177/1745691612469035

Farah, M. J., Hutchinson, J. B., Phelps, E. A., \& Wagner, A. D. (2014). Functional MRIBased Lie Detection: Scientific and Societal Challenges. Nature Reviews Neuroscience, 15(2), 123-31. https:/ / doi.org/10.1038/nrn3665

Farah, M. J., Smith, M. E., Gawuga, C., Lindsell, D., \& Foster, D. (2010). Brain imaging and brain privacy: A realistic concern? Journal of Cognitive Neuroscience, 21(1), 119127. https://doi.org/10.1162/jocn.2009.21010

Green, J. (2013). Moral Tribes: Emotion, Reason, and the Gap Between Us and Them. Penguin Press.

Grosenick, L., Greer, S., Knutson, B. (2008). Interpretable classifiers for FMRI improve prediction of purchases. IEEE Transactions on Neural Systems and Rehabilitation Engineering, 16(6), 539-548. https://doi.org/ 10.1109/TNSRE.2008.926701

Harris, J., Ciorciari, J., \& Gountas, J. (2018). Consumer neuroscience for marketing researchers. Journal of Consumer Behaviour, 17(3), 239-252.

https://doi.org/10.1002/cb.1710

Huppert, T., Barker, J., Schmidt, B., Walls, S., \& Ghuman, A. (2017). Comparison of group-level, source localized activity for simultaneous functional near-infrared 
spectroscopy-magnetoencephalography and simultaneous fNIRS-fMRI during parametric median nerve stimulation. Neurophotonics, 4(1), 015001. https://doi.org/10.1117/1.NPh.4.1.015001

Illes, J. (2007). Empirical neuroethics. Can brain imaging visualize human thought? Why is neuroethics interested in such a possibility? EMBO Reports, 8(1), S57-S60. https://doi.org/10.1038/sj.embor.7401007

Illes, J., Kirschen, M. P., \& Gabrielli, J. D. E. (2003). From neuroimaging to neuroethics. Nature Neuroscience, 6, 205. https:/ / doi.org/10.1038/nn0303-205

Jones, D. (2010). A WEIRD view of human nature skews psychologists' studies. Science, 328 (5986), 1627. https:// doi.org/10.1126/science.328.5986.1627

Kable, J. W. (2011). The cognitive neuroscience toolkit for the neuroeconomist: A functional overview. Journal of Neuroscience, Psychology, and Economics, 4(2), 63-84. https://doi.org/10.1037/a0023555

Karslake, J. S. (1940). The Purdue eye-camera: A practical apparatus for studying the attention value of advertisements. Journal of Applied Psychology, 24(4), 417-440. https://doi.org/10.1037/h0054171

Kenning, P., \& Plassmann, H. (2005). NeuroEconomics: An overview from an economic perspective. Brain Research Bulletin, 67(5), 343-354. https:// doi.org/10.1016/j.brainresbull.2005.07.006

Kenning, P., Plassmann, H., \& Ahlert, D. (2007). Applications of functional magnetic resonance imaging for market research. Qualitative Market Research: An International Journal, 10(2), 135-152. https:// doi.org/10.1108/13522750710740817

Knutson, B., \& Bossaerts, P. (2007). Neural antecedents of financial decisions. Journal of Neuroscience, 27(31), 8174-8177. https://doi.org/10.1523/JNEUROSCI.1564$\underline{07.2007}$

Kroeber-Riel, W. (1979). Activation research: Psychobiological approaches in consumer research. Journal of Consumer Research, 5(4), 240-250. https://doi.org/10.1086/208736

Krugman, H. E. (1971). Brain wave measures of media involvement. Journal of Advertising Research, 11(1), 3-9. http:/ / dx.doi.org/10.4135/9781452231501.n13

Kwong, K. K., Belliveau, J. W., Chesler, D. A., Goldberg, I. E., Weisskoff, R. M., Poncelet, B. P., Kennedy, D. N., Hoppel, B. E., Cohen, M. S., \& Turner, R. (1992). Dynamic magnetic resonance imaging of human brain activity during primary sensory stimulation. Proceedings of the National Academy of Sciences, USA, 89(12), 5675-5679. https://doi.org/10.1073/pnas.89.12.5675 
LaBarbera, P. A., \& Tucciarone, J. D. (1995). GSR reconsidered: A behaviour-based approach to evaluating and improving sales potency of advertising. Journal of Advertising Research, 35(5), 33-56.

Legrenzi, P., \& Umiltà, C. (2011). Neuromania: On the limits of brain science. Oxford University Press. https://doi.org/10.1093/acprof:oso/9780199591343.001.0001

Lindstrom, M. (2008). Buyology. Truth and Lies about why we buy. Random.

Madan, C. R. (2010). Neuromarketing: the next step in market research? Eureka, 1(1), 34-42. https:// doi.org/10.29173/eureka7786

Mañas-Viniegra, L., González-Villa, I. A., \& Llorente-Barroso, C. (2020a). The corporate purpose of Spanish listed companies: Neurocommunication research applied to organizational intangibles. Frontiers in Psychology, 11, e2108. https:// doi.org/10.3389/fpsyg.2020.02108

Mañas-Viniegra, L., Núñez-Gómez, P., \& Tur-Viñes, V. (2020b). Neuromarketing as a strategic tool for predicting how Instagramers have an influence on the personal identity of adolescents and young people in Spain. Heliyon, 6(3), e03578. https://doi.org/10.1016/j.heliyon.2020.e03578

McCabe, D., \& Castel, A. (2008). Seeing is believing: The effect of brain images on judgments of scientific reasoning. Cognition, 107, 343-352. https://doi.org/10.1016/j.cognition.2007.07.017

McClure, S. M., Li, J., Tomlin, D., Cypert, K. S., Montague, L. M., \& Montague, P. R. (2004). Neural correlates of behavioral preference for culturally familiar drinks. Neuron, 44(2), 379-387. https://doi.org/10.1016/j.neuron.2004.09.019

McClure, S. M., York, M. K., \& Montague, P. R. (2004). The neural substrates of reward processing in humans: The modern role of fMRI. Neuroscientist, 10(3), 260268. https://doi.org/10.1177/1073858404263526

Miller G. A., \& Lenneberg E. (1978). Psychology and biology of language and thought, essays in honor of Eric Lenneberg. Academic Press.

Mileti, A., Guido, G., \& Prete, M. I. (2016). Nanomarketing: A New Frontier for Neuromarketing. Psychology \& Marketing 33(8), 664-674.

https://doi.org/10.1002/mar.20907

Morin, C. (2011). Neuromarketing: The new science of consumer behavior. Society, 48(2), 131-135. https:/ / doi.org/10.1007/s12115-010-9408-1 
Moseley, M. E., \& Glover, G. H. (1995). Functional MR imaging. Capabilities and limitations. Neuroimaging clinics of North America, 5(2), 161-191. https://pubmed.ncbi.nlm.nih.gov/7640883

Mostafa, M. M. (2012). Brain processing of vocal sounds in advertising: A functional magnetic resonance imaging (fMRI) study. Expert Systems with Applications, 39(15), 12114-12122. https://doi.org/10.1016/j.eswa.2012.04.003

Nill, A., \& Schibrowsky, J. A. (2007). Research on marketing ethics: A systematic review of the literature. Journal of Macromarketing, 27, 256-273. https://doi.org/10.1177/0276146707304733

Nixon, H. K. (1924). Attention and interest in advertising. Archives of Psychology, 72, 5-67.

Nuñez, P. L., \& Srinivasan, R. (2006). A theoretical basis for standing and traveling brain waves measured with human EEG with implications for an integrated consciousness. Clinical Neurophysiology, 117(11), 2424-2435.

O'Connell, B., Walden, S., \& Pohlmann, A. (2011). Marketing and Neuroscience What Drives Customer Decisions? American Marketing Association. https://bit.ly/39zwWKM

Ohme, R., Matukin, M., \& Pacula-Lesniak, B. (2011). Biometric measures for interactive advertising research. Journal of Interactive Advertising, 11(2), 60-72. https://doi.org/10.1080/15252019.2011.10722185

Ohme, R., Reykowska, D., Wiener, D., \& Choromanska, A. (2009). Analysis of neurophysiological reactions to advertising stimuli by means of EEG and galvanic skin response measures. Journal of Neuroscience, Psychology, and Economics, 2(1), 2131. https://doi.org/10.1037/a0015462

Phelps, E. A., O'Connor, K. J., Cunningham, W. A., Funayama, E. S., Gatemby, J. C., Gore, J. C., \& Banaji, M. R. (2000). Performance on indirect measures of race evaluation predicts amygdala activation. Journal of Cognitive Neuroscience, 12, 729738. https://doi.org/10.1162/089892900562552

Plassmann, H., Ramsoy, T. Z., Milosavljevic, M. (2012). Branding the brain: A critical review and outlook. Journal of Consumer Psychology, 22, 18-36. https://doi.org/10.1016/i.jcps.2011.11.010

Plassmann, H., Venkatraman, V., Huettel, S., \& Yoon, C. (2015). Consumer neuroscience: Applications, challenges, and possible solutions. Journal of Marketing Research, 52(4), 427-435. https://doi.org/10.1509/jmr.14.0048 
Pynta, P., Seixas, S. A., Nield, G. E., Hier, J., Millward, E., \& Silberstein, R. B. (2014). The power of social television: Can social media build viewer engagement? A new approach to brain imaging of viewer immersion. Journal of Advertising Research, 54(1), 71-80. https://doi.org/10.2501/JAR-54-1-071-080

Racine, E., Waldman, S., Rosenberg, J., \& Illes, J. (2010). Contemporary Neuroscience in the Media. Social Science $\mathcal{E}$ Medicine, 71(4), 725-33. https://doi.org/10.1016/j.socscimed.2010.05.017

Rilling, J. K., \& Sanfey, A. G. (2011). The neuroscience of social decision making. $\begin{array}{lllll}\text { Annual Review } & \text { 23-48. }\end{array}$ https://doi.org/10.1146/annurev.psych.121208.131647

Roskies, A. L. (2015). Mind Reading, Lie Detection, and Privacy. In C. Clausen, \& N. Levy (eds.), Handbook of Neuroethics (pp. 679-695). Springer. https://doi.org/10.1007/978-94-007-4707-4_123

Russo, E. J. (1978). Eye Fixations Can Save the World: A Critical Evaluation and a Comparison Between Eye Fixations and Other Information Processing Methodologies. In K. Hunt, \& A. Abor (Eds.), Advances in Consumer Research (pp. 561-570). Association for Consumer Research.

Sanie, S., \& Chalmers, J. (2007). EEG signal processing. Chichester. Wiley.

Santesmases, M. (2009). Fundamentos de Marketing. Pirámide.

Santos, J. P., Seixas, D., Brandao, S., \& Moutinho, L. (2011). Investigating the role of the ventromedial prefrontal cortex in the assessment of brands. Frontiers in Neuroscience, 5, e77. https://doi.org/10.3389/fnins.2011.00077

Schimtt, F. O. (1970). Promising Trends in Neuroscience. Nature, 227, 1006-1009. https://doi.org/10.1038/2271006a0

Sententia, W. (2013). Freedom by design. In M. Moore, \& N. Vita-More (eds.), The transhumanist reader (pp. 355-360). Wiley-Backwell.

Smidts, A., Hsu, M., Sanfey, A., Boksem, M., Ebstein, R., Huettel, S., \& Yoon, C. (2014). Advancing consumer neuroscience. Marketing Letters, 25(3), 257-267. https://doi.org/10.1007/s11002-014-9306-1

Solnais, C., Andreu-Pérez, J., Sánchez-Fernández, J., \& Andréu-Abela, J. (2013). The contribution of neuroscience to consumer research: A conceptual framework and empirical review. Journal of Economic Psychology, 36, 68-81. https://doi.org/10.1016/j.joep.2013.02.011 
Spence, C. (2019). Neuroscience-Inspired Design: From Academic Neuromarketing to Commercially Relevant Research. Organizational Research Methods, 22(1), 275-298. https://doi.org/10.1177/1094428116672003

Spence, C., \& Piqueras-Fiszman, B. (2014). The perfect meal: The multisensory science of food and dining. Wiley-Blackwell.

Stanton, S. J., Reeck, C., Huettel, S. A., \& LaBar, K. S. (2014). Effects of induced moods on economic choices. Judgment and Decision Making, 9(2), 167-175. http://journal.sjdm.org/12/12924b/jdm12924b.pdf

Stanton, S. J., Sinnott-Armstrong, W., \& Huettel, S. A. (2017). Neuromarketing: Ethical Implications of its Use and Potential Misuse. Journal of Business Ethics, 144(4), 799-811. https:/ / doi.org/10.1007/s10551-016-3059-0

Tallis, R. (2011). Aping mankind: Neuromania, Darwinitis and the misrepresentation of humanity. Acumen Publishing.

Tiggemann, M., \& Zaccardo, M. (2016). Strong is the new skinny: A content analysis of \#fitspiration images on Instagram. Journal of Health Psychology, 23(8), 1003-1011. https://doi.org/10.1177/1359105316639436

Varan, D., Lang, A., Barwise, P., Weber, R., \& Bellman, S. (2015). How reliable are neuromarketers' measures of advertising effectiveness: data from ongoing research holds no common truth among vendors. Journal of Advertising Research, 55(2), 176-191. https://doi.org/10.2501/JAR-55-2-176-191

Venkatraman, V., Dimoka, A., Pavlou, P. A., Vo, K., Hampton, W., Bollinger, B., Hershfield, H. E., Ishihara, M., \& Winer, R. S. (2015). Predicting advertising success beyond traditional measures: New insights from neurophysiological methods and market response modeling. Journal of Marketing Research, 52(4), 436452. https://doi.org/10.1509/jmr.13.0593

Vul, E., Harris, C., Winkielman, P., \& Pashler, H. (2009). Puzzlingly high correlations in fMRI studies of emotion, personality, and social cognition. Perspectives on Psychological Science, 4(3), 274-290. https://doi.org/10.1111/j.1745$\underline{6924.2009 .01125 . x}$

Wedel, M., \& Pieters, R. (2008). A review of eye-tracking research in marketing. Review of Marketing Research, 4, 123-147. https://doi.org/10.1108/S1548$\underline{6435(2008) 0000004009}$

Yan, L. (2011). An Analysis of the Research Status of Neuromarketing. Foreign Economics \& Management, 33(5), 25-27. 
Lyu, D., y Mañas-Viniegra, L.

Problemas éticos en la investigación con neuromarketing: una revisión de la literatura

Zaltman, G., \& Kosslyn, S. M. (2000). Neuroimaging as a marketing tool. US Patent $6,099,319$.

Zurawicki, L. (2010). Neuromarketing. Exploring the Brain of the Consumer. Springer

\section{AUTOR/ES:}

\section{Dongye Lyu}

Doctorando en Comunicación Audiovisual, Publicidad y Relaciones Públicas por la Universidad Complutense de Madrid, es asistente de investigación en Tongji University (China). Sus intereses de investigación son los estudios de comunicación en el ámbito del deporte.

Research Gate: https://www.researchgate.net/profile/Dongye_Lyu

\section{Luis Mañas-Viniegra}

Doctor en Comunicación Audiovisual y Publicidad con Premio Extraordinario, es Profesor Contratado Doctor interino en la Universidad Complutense de Madrid e IP del grupo de investigación UCM Brand Lab, actualmente en validación.

Orcid ID: https:/ / orcid.org/0000-0001-9129-5673

Google Scholar: https://scholar.google.es/citations?user=UsVJTKUAAAAJ\&hl=es

Scopus: https:// www.scopus.com/authid/detail.uri?authorId=57197747570

Publons: https://publons.com/researcher/1396662/luis-manas-viniegra/

Research Gate: https://www.researchgate.net/profile/Luis_Manas-Viniegra 